\title{
Electro-optics communication course for electronics engineering students
}

Aaron Peled

Aaron Peled, "Electro-optics communication course for electronics engineering students," Proc. SPIE 1603, Education in Optics, (1 March 1992); doi: $10.1117 / 12.57856$ 
Electrooptics communication course for electronic engineering students

Aaron Peled

Electrical and Electronics Department, Center for Technological Education Holon (CTEH), aff. Tel-Aviv University (TAU), 52 Golomb Street, Holon 58102, Israel

\section{ABSTRACT}

The scope of this paper is to present a specific program of an Electrooptics Communication course taught for ten years during the final year at undergraduate level at CTEH. Our interpretation of the subjects to be given in such a course is to supplement optical concepts and integrate them with previously taught basic principles in telecommunication courses, physics and electromagnetic theory. This means that the course relies upon former knowledge of electronic engineering of old established communication technologies, such as microwave radio, coaxial transmission, wire-pair data communication systems and telecommunication theory. In this way, EE students receive a condensed but balanced integration of EO communication concepts from the various disciplines. These elements allow them to design and implement in their final year, practical projects of Electrooptic communication circuits and systems, which excell today in device availability and economics.

\section{INTRODUCTION}

Electrooptics (EO) Communication integrates Optics, Electromagnetic (EM) Radiation in the visible (VIS) to infrared (IR) and electronics. Advances in all the abovementioned areas, during the past decade, have contributed to the emerging of the EO communication electronics industry. NOw this industry demands engineers and circuit designers with basic training and knowledge of the principles and techniques involved in this relatively new branch of electronics related technology. However, since electronics engineering (EE) curricula have not included a mandatory engineering treatment of this subject in the past, many generations of industry electrical and electronic engineers lack even basic knowledge of many aspects of this discipline. This happened because many EE students could not attend dedicated courses in EO, mainly due to other priority constraints imposed on them by the regular undergraduate $\mathrm{EE}$ courses. However, the time has now come when a strong demand is felt for a basic course which integrates enough-optics and electronics to be merged with techniques taught in the general EE curricula. This process happened over the past several years during which optical communication systems became compatible with, or even surpassed, traditional electronic oriented communication systems in several applications, such as long haul telephony and computer networks. In fact, even in the professional journals devoted in the past to telecommunication techniques and theories, we have witnessed a tremendous shift of published papers from radio, microwave $(\mu W)$ and wired links to the newly E0 communication subjects. However, this is of no big surprise since information processing and channel capacity have increased in electronics to the range beyond $\mathrm{GHz}$ frequency. This trend created a tremendous pressure for opening up new modulation bands at higher frequencies which are natural to optical carriers. Obviously, one cannot hope to cover all aspects of E0 communication in a one semester introductory course, although many excellent texts have appeared in the literature during the past decade. ${ }^{1-19}$ Hence, this course was designed to fit in the regular $\mathrm{EE}$ courses relying on former studies, 
concentrating mainly upon integration of old technologies concepts with the specific parameters of optical elements and optical EM radiation. To our aid comes the fact that in a well balanced EE curricula, students are exposed to all basic theories needed to understand an engineering based treatment of EO communication. For example, the old established comunications technologies, i.e. microwave links and coax cables, have basically common parameters to optical communication such as EM wave propagation, channel frequency allocation, insertion losses, delays, bandwidth, noise concepts, analog and digital modulation, etc. Hence, in the preparation of such a time-material limited course, one has merely to show how the decade old established EO technology supplies very efficient methods to implement electronically driven optical transmitters, detectors and fiber media.

We also found it very satisfying to realize that some students who attended the course became interested in this area and were able to implement easily practical projects on optical communication in their $4^{\text {th }}$ year final project. This east of project making stands in contrast to the regular telecommunication projects which are much more demanding in complexity, device availability and economics. Some typical projects are given in Refs.(20-22). In a way, this course is quite advanced by requiring many prerequisite courses, but on the other hand, it is still at an introductory level for someone who wishes to specify more in this field. Hence it obviously fills the introductory needs of undergraduate EE students only. In the following sections, we present the basic subjects of the course developed and taught at CTEH for a decade. It implements the former mentioned concept of integrating optical physics, microwaves and telecommunication theory to obtain a basic introductory EO comminication course, for $\mathrm{EE}$ students.

\section{INTRODUCTION TO OPTICAL COMMUNICATION}

This chapter provides an introduction to EO communication by way of considering recent channel capacity needs, mainly for audio, video and computer data transmission. Using Shannon's formula ${ }^{8}$ for channel capacity:

$$
C=B W \cdot \log _{2}(1+S / N),\left(\frac{\text { bits }}{\sec }\right)
$$

it is illustrated by several examples that coherent modulation techniques at optical frequencies may provide a tremendous increase (by a factor of $10^{6}$ ) of the rate of information transmission, as compared to existing electronics based telecommunication. In this way, EE students appreciate mostly the impetus for opening up the Terahertz frequency band to the already clogged electronic communication traffic. An example shows, for instance, how to multiplex theoretically $10^{6}$ video channels over a single communication path. Another example relates to the extremely low loss and small size of optical fiber links as compared with various metallic waveguiding media such as coax cable and circular waveguides. This being the result of the different loss mechanisms, bulk vs. surface resistive dissipation of the two technologies, see Fig.1. Fig.1 also emphasizes the higher capacities available for FO waveguides, which can satisfy more easily the bandwidth "hungry" digital communication systems relation:

$$
B W_{\text {system }} \geqslant 2 \cdot B W_{\text {channel }} \cdot n_{\text {channels }} \cdot N_{\text {samples }}
$$




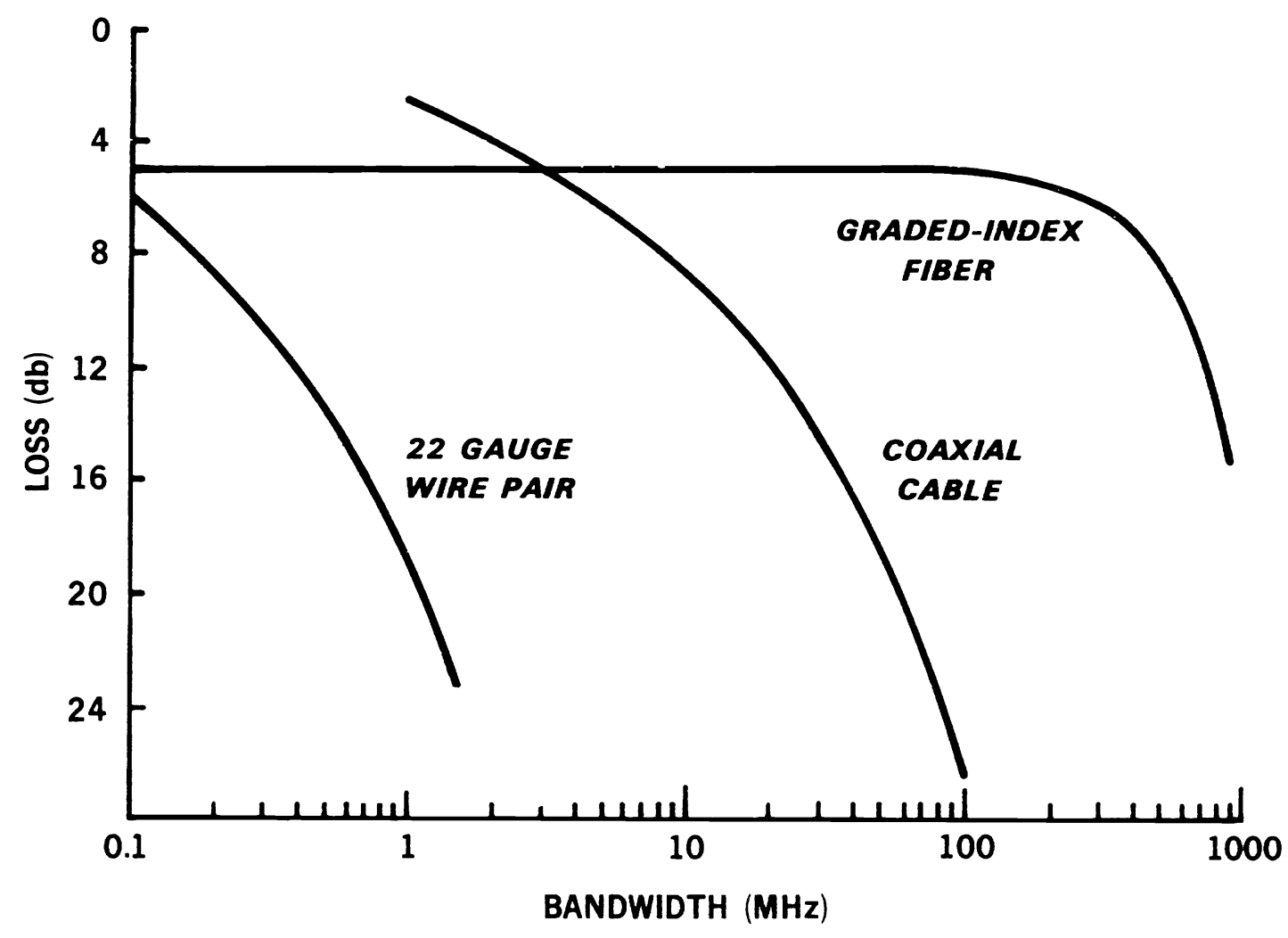

Fig.1: Effective Loss of $1 \mathrm{~km}$ Lengths of Transmission Media

3. ELECTROMAGNETIC WAVE PROPAGATION IN MEDIA

This chapter gives a theoretical treatment of the EM wave phenomena. It relies upon previous electromagnetism or microwave courses which are usually given to EE students in the second or third year of their curricula. The basic properties of the planar and radial wave equations are reviewed ${ }^{18}$ with emphasis on optically oriented concepts such as:

$$
\begin{aligned}
& \text { Photon Energy and wave vector : } E=h f ; \quad k=2 \pi / \lambda \\
& \text { Complex Optical Index: } \quad: N=n-j k \\
& \text { Dispersive media: } \quad: k=\frac{\omega}{c} n(\omega) \\
& \text { Phase and Group Velocities } \quad: v_{p}=\lambda f ; v_{g}=\frac{d \omega}{d k}=\frac{c}{n+\omega \frac{d n}{d \omega}} \\
& \text { Material Group Refractive Index: } n_{g}=n+\omega \frac{d n}{d \omega} \\
& \text { Group Delay } \\
& : \tau_{d}=1 / v_{g}\left(\frac{\mathrm{sec}}{\mathrm{km}}\right)
\end{aligned}
$$


Several exercises are given to get the feeling for quantities involved in the calculation of group velocities and group dispersion times, for real life fiber optic (FO) media and atmospheric communication paths. The difference between the radio oriented plane wave description of EM waves, as compared to typical optical point sources is discussed. Finally the continuum approach relating Radiant Flux Density of the EM field to the time average of Poynting Vector is taught by calculations with equations such as:

$$
\langle S\rangle_{a v}=\frac{1}{2} C \varepsilon E_{0 z}^{2}\left(\text { watt } / \mathrm{m}^{2}\right)
$$

\section{POLARIZATION OF THE EM WAVES - PASSIVE AND ACTIVE COMPONENTS}

It is natural to bring this concept immediately after Chapter 2, since EM basics allow straightforward definition of polarization of the electric field vector. The more basic reason, however, for introducing polarization in this course is to show that it is a fundamental method for modulating EO sources, which cannot be driven directly electronically. Polarization effects are unified conceptually through the difference in refraction index:

$$
\Delta \mathrm{n}=\mathrm{n}_{n}-\mathrm{n}_{\perp}
$$

Then the following classification follows:

(a) Passive Polarizers:

Dichroic (Malus Law) : $E_{"}-\left|E_{0}\right| \cos \theta$

Reflective (Brewster Angle): $\theta_{B}=\operatorname{arctg}\left(\frac{n_{t}}{n_{i}}\right)$

Birefringe : $\Delta n=n_{o x}-n_{o r} ;$ Ord/Extraordinary refraction

(b) EM Activated Polarizers: $\Delta n=\alpha+\beta F+\gamma F^{2}+\ldots$

Kerr Effect $: \Delta n \simeq \gamma|\bar{E}|^{2}$

Pockel Effect : $\Delta n \simeq \beta|\bar{E}|^{2}$

Faraday Effect: $\Delta n \simeq \gamma|\bar{B}|^{2}$

A typical treatment of an EO modulator operation 7,8 based on the Pockel Effect is given: 


$$
\Delta \mathrm{n}= \pm \frac{1}{2} \mathrm{rn}_{0}^{3} \mathrm{E}_{\mathrm{z}}
$$

AM and PM modulation treatments by changing the light intensity with an externally imposed voltage $v_{z}$ on the $\mathrm{EO}$ cell is given:

$$
I_{m \circ d}\left(V_{z}\right)=2 E_{0 z}^{2} \sin ^{2}\left(\frac{\pi}{2} \cdot \frac{\omega}{c} \cdot n_{0}^{3} \cdot r \cdot V_{z}\right)
$$

\section{INTERFERENCE THEORY REVIEW}

Diffraction and Interference, being extremely important to the resolving power and coherence concepts in optical communication and LIDAR systems, are reviewed. Diffraction is also important to understand the principles of specific EO modulators. Diffraction is reviewed by the general superposition method of complex EM waves emanating from a linear aperture $b=\mathrm{N} \cdot \mathrm{a}$

$$
E_{c}(P)=A(r) \cdot e^{-j \omega t} \cdot \sum_{i=1}^{N} e^{j k r_{i}}
$$

leading to the sinc diffraction function:

$$
E_{r}(r, \theta)=N \cdot A(r) \cdot \operatorname{sinc}\left(\frac{\pi \cdot a \sin \theta}{\lambda}\right)
$$

with a periodic space pattern:

$$
m \cdot \lambda=b \cdot \sin \theta \quad ; \quad m=0, \pm 1, \pm 2, \ldots
$$

From the diffraction condition, the angular and spatial resolving power concepts are derived for optical telescopes and $\mu \mathrm{W}$ dishes:

$$
\theta_{\mathrm{diff}} \simeq \lambda / \mathrm{b} \quad ; \quad \mathrm{w}_{\mathrm{diff}} \simeq 2 \cdot \mathrm{x} \cdot \lambda / \mathrm{b}
$$

Interference is treated as a generalized diffraction array leading to the diffraction grating-equation:

$$
I_{o p t} \simeq A_{0}^{2} \cdot \frac{\sin ^{2} \beta}{\beta^{2}} \cdot \frac{\sin ^{2}(N \gamma)}{\sin ^{2} \gamma}
$$

Coherence is treated in this chapter by way of analyzing the Fourier Transform of a time restricted sinusoidal pulse of duration $\Delta t_{c o h}$. The result, again being a time-space sinc function gives for the first lobe zero condition:

$$
\left(\omega_{0}-\omega\right) \cdot \Delta t_{c \circ h} \simeq 2 \pi
$$

which is the simplest way of defining temporal and length coherence of a light source. Several examples of various lasers are given to provide real life problems. The spatial coherence concept, lateral and longitudinal is then discussed to show the important directivity feature of lasers. This being useful in LIDARS and Laser material processing systems. 


\section{OPTICAL ENGINEERING DESIGN}

EE students cannot usually devote much of their time to all the aspects of classical optical engineering. However, we believe they must acquire some optical engineering oriented basics at a higher than elementary optical physics level to communicate better with optical systems design engineers. With this purpose in mind, the basic reflection and refraction rules are shown to generalize the basic equations for beam shaping elements. The elements discussed are mirrors, corrected lens assemblies and the optical fiber basis. The following relations and parameters are reviewed in connection with collimation, converging and diverging of beams:

$$
\mathrm{p}^{\prime}=\mathrm{p} \cdot \frac{\mathrm{f}}{\mathrm{p}-\mathrm{f}}=\mathrm{pM}=\mathrm{f}(\mathrm{M}+1)
$$

Light collection efficiency is introduced by the Numerical Aperture and $f^{*}$ number of macro and micro optical systems:

$$
\mathrm{f}^{\#}=\mathrm{f} / \mathrm{D} \quad ; \quad \mathrm{NA}=\mathrm{n}_{0} \cdot \sin \theta \quad ; \quad \mathrm{NA} \infty \frac{1}{2 \mathrm{f}^{*}}
$$

For the communication important fiber optic element, the NA expression is developed:

$$
N A \simeq \sqrt{n_{1}^{2}-n_{2}^{2}}
$$

Two real life exercises are given in which one deals with the design of a typical optical projection system, the other relating to field lens zooming for radiometry. In this way, EE students are introduced to practical optical system design problems of transmission and receiving of EM radiation in the IR and VIS range for imaging and communication purposes.

\section{RADIOMETRY AND PHOTOMETRY}

In this chapter, the EE student is made familiar to the two parallel approaches of Radiometry and Photometry. One is for quantifying EM radiation in the whole range from $\mu W$ through IR to VIS and UV, while photometry is a historically older subset of the former. To avoid excessive distraction of EE students from the important issues, only four energy based concepts are defined and used in this course:

$$
\begin{aligned}
& \text { (a) } \mathrm{P}=\text { Radiant Flux/Spectral Radiant Flux; } \mathrm{W} ; \frac{\mathrm{W}}{\mu \mathrm{m}} ; \text { Lumen } \\
& \text { (b) } \mathrm{E}=\text { Radiance and Exitance/Spectral } ; \frac{\mathrm{W}}{\mathrm{m}^{2}} ; \frac{\mathrm{W}}{\mu \mathrm{m} \cdot \mathrm{m}^{2}} ; \mathrm{Lux} \\
& \text { (c) } \mathrm{J}=\text { Radiant Intensity of Source ; } \frac{\mathrm{W}}{\mathrm{S}_{\mathrm{r}}} ; \text { Candela } \\
& \text { (d) } \mathrm{N}=\text { Radiance of source } ; \frac{\mathrm{W}}{\mathrm{Sr} \cdot \mathrm{m}^{2}} ; \text { Candela } / \mathrm{m}^{2}
\end{aligned}
$$


The conversion of photometric to radiometric units and vice versa is given through the CIE Standard Visibility Function, $V(\lambda)$ and the following integral:

$$
P_{L}=K_{m} \int_{280}^{760} V(\lambda) \cdot P_{0 \lambda}(\lambda) d \lambda
$$

This chapter also introduces the following Geometry/Power relations of various sources:

(a) Isotropic point source: $P=J \cdot \omega ; J(\theta)=$ constant

(b) Spherical source : $P=2 \pi \cdot N(0) \cdot A$

(c) Lambertian source : $P=\pi N(0) \cdot A=\pi J(0) ; J(\theta)=J(0) \cos \theta$

For most laser sources, the following stereometric angles of exitance relation is given:

$$
\Omega=2 \pi(1-\cos \theta) \simeq \pi \theta^{2} \quad \text { (steradians) }
$$

Several worked examples are given to develop the Isotropic and Lambertian concepts for light sources. The LIDAR equation is developed, and used as an example:

$$
P_{R E C}=P_{T R} \cdot\left(\frac{\theta_{3} \cdot \theta_{2}}{\theta_{1}}\right)^{2} \cdot \eta_{A} R_{1}+R_{2} \cdot \rho_{0} \cdot p \text { (watt) }
$$

Also a full design of a Fiber-optic illuminator system is worked out to show integration of concepts such as optical microprojection, fiber coupling, optical shaping of the output beam and radiometry.

\section{SOURCES IN THE UV-VIS-IR EM REGION}

Light sources are classified here according to the three main classes: Blackbody, Incoherent Luminiscent and Lasers. The Blackbody radiation equation is used to remind the students the continuous spectra sources:

$$
W(T)=\int_{\lambda_{1}}^{\lambda_{2}} W(\lambda, T) d \lambda \quad ; \quad W(\lambda, T)=\frac{E(\lambda) \cdot C_{1}}{\lambda^{5}\left(e^{C_{2} / T}-1\right)}
$$

Stephan-Boltzman and wien Shift Laws are reviewed for typical incandescent light sources to be discussed later with the greenhouse effect, thermal imaging and Forward Looking IR (FLIR) systems. Then the quantum luminiscent sources are introduced by the typical Luminiscent Injection Diode (LED). The direct gap semiconductor sources, GaAs GaP, GaAsP and SiC which cover the whole IR-VIS spectrum are described and their quantum shaped spectra. The practical optoelectronic parameters such as: Optical Power vs. Diode Current and wavelength, I-V characteristics and Spatial Intensity distribution are taught and exercised. Basic electronic driving circuits for LED's are given for analog and digital applications. Then a special section is devoted to Laser 
sources. We classified them as follows: Gas Lasers $\left(\mathrm{CO}_{2}\right.$, HeNe and Ion), Chemical Lasers (HF:DF), Dye Lasers (Pumped- $\mathrm{N}_{2}$, Excimer, Nd:YAG), Tunable Excited Monomer ( $\mathrm{ArF}, \mathrm{XeF}, \mathrm{XeCl}, \mathrm{KrF}$ ), Solid State Lasers (Ruby, Na:YAG) and finally the most important to communication, semiconductor lasers (GaAs, GaAlAs, InGaAsP). The physical mechanism of LASER operation is reviewed emphasizing the basic conditions for lasing action: Spontaneous and Stimulated Emission, Optical pumping in gases and solid state lasers, electrical injection pumping in semiconductor lasers and population invertion. Finally the resonant cavity conditions for typical gas solid state and semiconductor lasers ${ }^{16}$ are calculated. This chapter ends with the description of optoelectronic typical characteristics of semiconductor lasers and two analog electronic driving schemes.

\section{DETECTORS FOR RADIATION}

The detector function is identified as a transducer function from EM radiation to electrical signals, : voltage, current and power. Responsivity, Quantum Efficiency and wavelength are related by the basic photoelectric conversion formula ${ }^{6}, 11$ :

$$
R(\lambda)=\frac{\lambda}{h c} \cdot n \cdot q_{0}
$$

The Noise Concept used is Noise Equivalent Power (NEP) and its closely related Minimum Detectable Signal:

$$
N E P=\frac{P_{R E C}}{\sqrt{B W} \cdot\left(\frac{S}{N}\right)}
$$

The definition of Detectivity is also given to allow some limited treatment of IR detectors used in thermal imaging and FLIR. Three classes of detectors are discussed: Non-quantum detectors (Thermocouple, TCR and Gas Expansion), quantum detectors (photoemission, photojunction, photovoltaic and photoconductor) and photoelectric cells. The treatment for pn junction photodetectors is more extensive starting from basic concepts such as photocurrent models:

$$
I_{p h}=R(\lambda) \cdot P_{R E C}
$$

The basic optoelectronic characteristics are given for the photo-diode. The basic I-V-P $P_{R E C}$ characteristic is shown with emphasis on the photoconductive and photovoltaic regions proper biasing with simple detection circuits. Other parameters which are discussed are Dark Current and photodiode capacitance. The equivalent electrical circuit of the photodiode is given and the elemental limiting noise sources, thermal and quantum-shot are exercised:

$$
N E P=\frac{I_{n}}{R(\lambda)}=\frac{1}{R(\lambda)} \sqrt{2 q_{0} \cdot I_{D} \cdot B W+\frac{4 k T}{R_{L}} \cdot B W}
$$

Transition regimes from thermal to shot noise limited circuits are calculated. Then response times and electrical bandwidth of the photodiode circuit is 
developed by the dominant pole approximation ${ }^{11}$ :

$$
t_{R} \simeq 2.2 \cdot R_{L} \cdot C_{D} \quad ; \quad B W_{-3 d B} \simeq \frac{0.35}{t_{R}}
$$

The various recent photodiode technologies are discussed in view of their relative merits: PIN, ADP, phototransistor, photovoltaic cells and photoresistors (CdS, CdSe, PbS, PbSe). This chapter is concluded with extensive design and analysis of biasing of detector-amplifier circuit combinations allowing the future electronic engineers to acquire some experience in submicrovolt electronics design. Circuits suitable for both analog and digital receiving systems are given, see example in Fig.2:

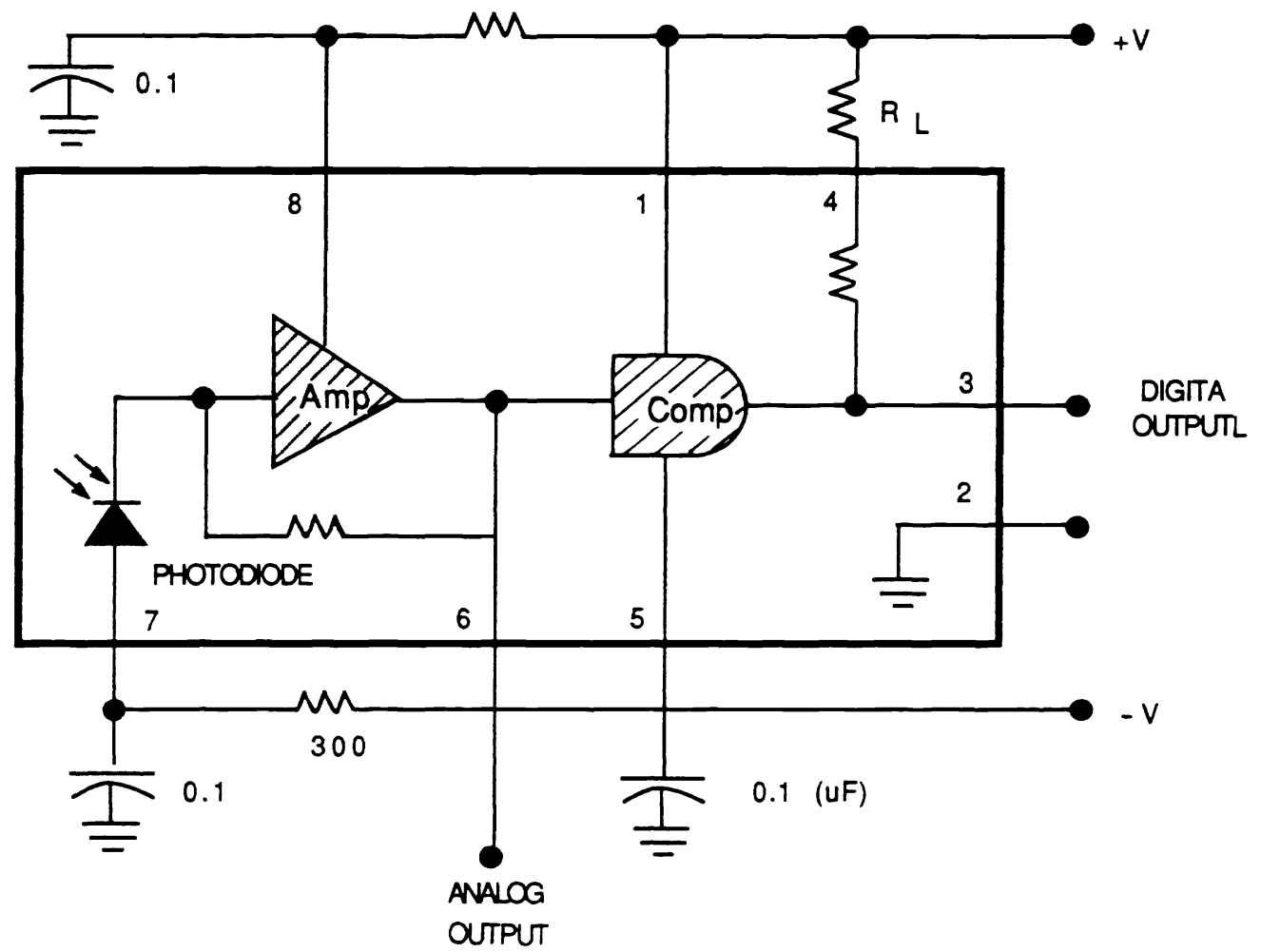

Fig.2: ANALOG/DIGITAL Receiver Circuit

10. EO COMMUNICATION SYSTEM AND MODULATION FORMATS

This chapter integrates telecommication engineering concepts with the electrooptic energy conversion elements discussed in the previous chapters. Analogous to any other communication system, EO comunication systems can be divided into two groups, guided (FO) and unguided (Line of Sight Communication or LIDAR). Also, there is a transmitter where the information is encoded, imposed upon some EM carrier and coupled to a transmission media. Finally, the receiver end abstracts the information from the received optical power. The type of information and its implementation by the various modulation schemes, be it digital or analog, are reviewed from former communication courses. The important feature that today's optic baseband transmission is obtained by the rather simple Intensity Modulation (IM) scheme is discussed and compared with the classical AM modulation of radio frequency carriers. In essence, AM 
modulation shifts the baseband coherently to a new EM region, which is, however, a very complicated task in the optical frequencies band. Since for most semiconductor optic transmitters, the optical power varies proportionately to the input current, we can still implement AM/IM modulation which generates incoherent modulated optic power of coherently radio or microwave AM modulated information:

$$
P_{o p t}(t)=P_{0}+P_{s}\left(1+m \cos \left(\omega_{o p t} t\right) \cdot \cos \left(\omega_{s c} \cdot t\right)\right.
$$

With $\omega_{s} \ll \omega_{o p t}$ it gives rise to the so-called subcarrier modulation ${ }^{1,16}$. Also, the same applies to FM/IM and PM/IM optical subcarrier modulations. Digital modulation formats are also recalled, emphasizing the good compatibility of optical transmitters to modulation codes, such as: PCM, PPM and PDM. As is the case with analog subcarrier modulation, ON/OFF keying of subcarriers can be implemented to obtain FSK, PSK and other digital coding schemes.

Digital modulation also allows subcarrier frequency-division and time multiplexing ${ }^{16}$. Moreover, it is emphasized that as long as optical devices have enough frequency response, the optical communication system can be used as a "transparent" communication device for almost any other digital modulation scheme such as delta-modulation, for instance. Today, however, still the most attractive choice for many fiber systems is PCM, due to transmitter and receiver simplicity of design. Optical Heterodyne is a subject touched only lightly in this course, since today's systems are still on the research laboratory benches.

\section{FIBER OPTICS COMMUNICATION SYSTEMS (LINRS)}

This is the last but most significant chapter, since it is devoted entirely to the design and implementation of a general type Fo communication link. It relies heavily upon the former chapters dealing with the design of circuits for optical Transmitters and Photodiode detection circuits. The chapter starts with the classification of the various fibers currently in use: step, monomode and graded multimode. The basic equations specifying the fiber characteristics are given:

$$
\begin{aligned}
& \text { Number of Modes }=\frac{2 \pi}{\lambda^{2}}(\text { Core Area }) \cdot(N A)^{2} \\
& \text { Power Coupling into Fiber }=\left(\frac{\pi D}{2}\right)^{2} \cdot N(0) \cdot(N A)^{2} \\
& \text { Power Coupling to detector }=P_{F} \cdot \frac{1}{\pi d^{2}(N A)^{2}}
\end{aligned}
$$

$$
\text { Fiber Attenuation }(d B)=10 \log _{10}\left(P_{I N}^{O P T} / P_{O U T}^{O P T}\right)
$$

Insertion Losses (IL) throughout the system are dealt with such as: interface coupling, splices and connectors: 


$$
I L=L * \cdot X+\sum_{j=1}^{K} L_{j}
$$

Finally the three interdependent design equations of required receiver power for a specific information data rate capacity or analog bandwidth are discussed ${ }^{10}$ :

$$
\begin{gathered}
B W_{s y s t}=\frac{P_{R E C}^{n}}{k \cdot T_{s y s t}\left(\frac{S}{N}\right) \cdot E N F} ; n=1 \text { : large signal } \\
P_{R E C}=P_{T R}(d B m)-I L(d B) \\
X_{m a x}=\frac{P_{T R}(d B m)-P_{R E C}(d B m)-I L(d B)}{L \star(d B / k m)}
\end{gathered}
$$

where $\mathrm{x}_{\max }$ is the maximum repeatless link length. Finally, the overall system Bandwidth ${ }^{\text {ax }}$ considerations are given through the cascaded approach of transmitter, fiber and detector components.

$$
T_{\text {system }}^{\text {risetime }}=\sqrt{T_{\text {Fiber }}^{2}+\left(\frac{0.35}{B W_{R E C}}\right)^{2}+T_{T R}^{2}}
$$

or:

$$
B W_{\text {system }} \simeq\left[\left(\frac{1}{B W_{F i b e r}}\right)^{2}+\left(\frac{1}{B W_{R E C}}\right)^{2}+\left(\frac{1}{B W_{T R}}\right)^{2}\right]^{-1 / 2}
$$

where the Fiber model equations for Bandwidth are obtained from modal and group dispersion:

$$
\begin{aligned}
& B_{F i b e r}=\frac{K}{\sqrt{D_{1}^{2}+D_{2}^{2}}} \\
& D_{1} \simeq \frac{1}{c} \cdot \lambda \cdot\left(\frac{d^{2} n}{d \lambda^{2}}\right) \cdot \Delta \lambda \\
& D_{2} \therefore \frac{n}{c} \cdot \frac{1}{2}\left(\frac{N A}{n}\right)^{2}
\end{aligned}
$$

Fiber Bandwidth

group dispersion

modal dispersion

and bandwidth link/length dependence ${ }^{10}$ through: 


$$
\mathrm{BW}_{\text {Fiber }}(\mathrm{x}) \simeq \mathrm{BW}(1 \mathrm{~km}) / \mathrm{X}^{\mathrm{n}} ; \quad \mathrm{n}=0.5-1.0
$$

\section{CONCLUSIONS}

In this paper, I have reviewed and emphasized the main subjects given in an Electro-optic communication course of introductory level for EE undergraduate students. Since there is no doubt that guided and unguided EO systems will be extensively used in telecommunication and data transmission systems, a basic training such as suggested in this paper is obviously needed. Extracting the relevant subjects from this vast field is, of course, the lecturer's choice. However, from the electronics engineering point of view, teaching this subject poses a great dilemma, since, although good texts and books are available ${ }^{1-19}$, they are too extensive to be given in a one semester course. Hence, what we have in mind is a course which condenses all the practically relevant subjects needed to achieve a basic understanding of EO comunication principles. From ten years of experience in teaching these subjects, virtually as described in this paper, we can say that the results were quite promising. We found it very satisfying to realize that of the regular EE students who attended the course, about $10 \%$ became interested and performed their $4^{\text {th }}$ year final practical project in this area.

Some typical projects subjects are given in Refs. $(20,21,22)$. Finally, what we would like to stress is that although this course seems to be of an introductory level, in fact, it is much more advanced since it requires a lot of prerequisite courses in telecommunication and electromagnetism. Hence, although it obviously fills the needs of undergraduate EE students, it is certainly not designed for students from other disciplines with different curricula.

\section{REFERENCES}

1. A. Chappel, "Optoelectronics - Theory and Practice", Texas Instruments Edition, NY, 1976.

2. J.E. Midwinter, "Optical Fibers for Transmission", John Wiley, NY, 1979.

3. M.J. Howes and D.V. Morgan, Eds., "Optical Fibre Communications: Devices, Circuits and Systems", John wiley, Chichester, 1980.

4. C.P. Sandbank, Editor, "Optical Fibre Communication Systems", John Wiley, Chichester, 1980.

5. M.K. Barnoski, "Fundamentals of Optical Fiber Communications", Academic Press, NY, 1981. 1981.

6. S.D. Personick, "Optical Fibre Transmission Systems", Plenum Press, NY,

7. J. Wilson and J.F.B. Hawkes, "Optoelectronics: An Introduction", Prentice Hall Intl., Englewood Cliffs, 1983.

8. J. Gowar, "Optical Communication Systems", Prentice Hall Intl., Englewood Cliffs, 1984 .

9. A. Yariv, "Optical Electronics", Holt-Reinhardt, NY, 1985. 1984.

10. G. Keiser, "Optical Fiber Communications", McGraw Hill Intl., Auckland,

11. J. Senior, "Optical Fiber Communication: Principles and Practice", Englewood Cliffs, 1985. 1986.

12. M. Tischler, "Optoelectronics: A Text-Lab Manual", McGraw Hill, NY, 
13. P. Halley, "Fibre Optic Systems", John-Wiley, Chichester, 1987. 1987.

14. G. Mahlke and P. Gosing, "Fiber Optic Cables", John-Wiley, Chichester,

15. M. Katzman, Editor, "Laser Satellite Communications", Prentice Hall, Englewood Cliffs, 1987.

16. J.C. Palais, "Fibre Optic Communications", Prentice Hall, Englewood Cliffs, 1988.

17. H.B. Killen, "Digital Communication with Fibre Optic and Satellite Applications", Prentice Hall, Englewood Cliffs, 1988.

18. A. Ghatak and K. Thyagarajan, "Optical Electronics", Cambridge University Press, Cambridge, 1989.

19. R.G. Seippel, "Optoelectronics for Technicians and Engineering", Prentice Hall, Englewood Cliffs, 1989.

20. List of CTEH Final year undergraduate practical projects: G. Zimermann, "Computer Programs for Design of FO Digital Communication Systems, 1985; A. zentner, "Design of an Electrooptic Microphone with high bandwidth", 1985; A. Levy, "DC/DC Converter of 5-100 (V) for Optoelectronics Low Current Output, 1986; S. Argoetti, "Digital and Analog Fiber Optics Communication System Design and Bandwidth Optimization", 1986; M. Huper and L. Zvi, "Investigation of Software Algorithms for Digital Image Processing", 1987; N. Katz and D. Krauss, "Transmitter/Receiver implemented by EO modules, 1987; Z. Yacov and N. Kalmi, "Investigation of Bandwidth/Noise Concepts in Distributed FO Systems", 1987; D. Kolpinitzki, "Design and implementation of an FM/IM Line of Sight EO Communication System", 1987; M. Malka and M. Petel, "Data FO Transmission System in Noisy Environments", 1988; H. Aharoni, "Design and Implementation of an FO Modem for Communication on IBM PC systems, 1990; N. Sofer and I. Hashfar,, "An Electrooptic Communication System", 1990; S. Bason, "Design and Implementation of a driver for Laser Diodes, 1990; Y. Kamil, "Calculations of FO Communication Links, 1990; V. Geuli, "Design and Implementation of an Optical Reader", 1991.

21. A. Peled and S. Argoetti, "Low Power FO Digital Communication Links by Pulsed Unipolar Marking Code", Intl. J. of Optoelectronics, $\underline{3}(5)$, pp.439-442, 1989.

22. A. Peled, S. Argoetti and J. Gavan, "Channel Capacity Enhancement in Segmented FO Data Communication Links", Intl. J. of Optoelectronics, $\underline{4}(1)$, pp.63-68, 1989.

\section{APPENDIX I: GLOSSARY OF SYMBOLS}

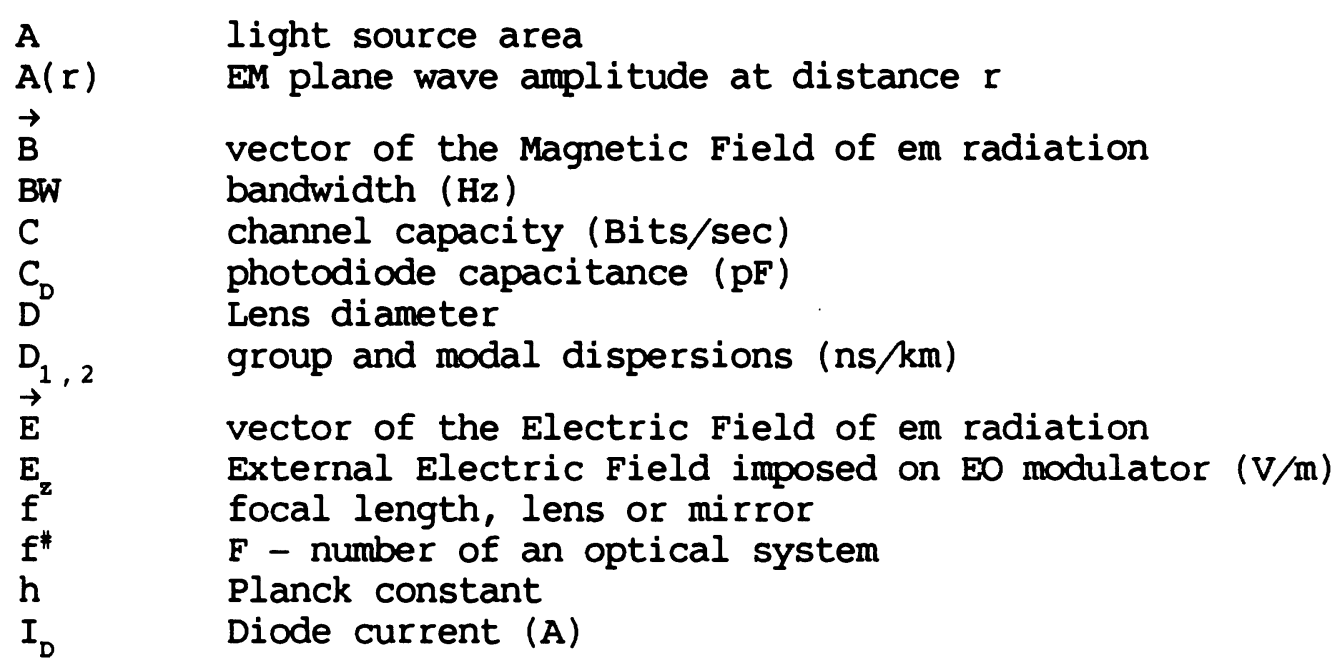




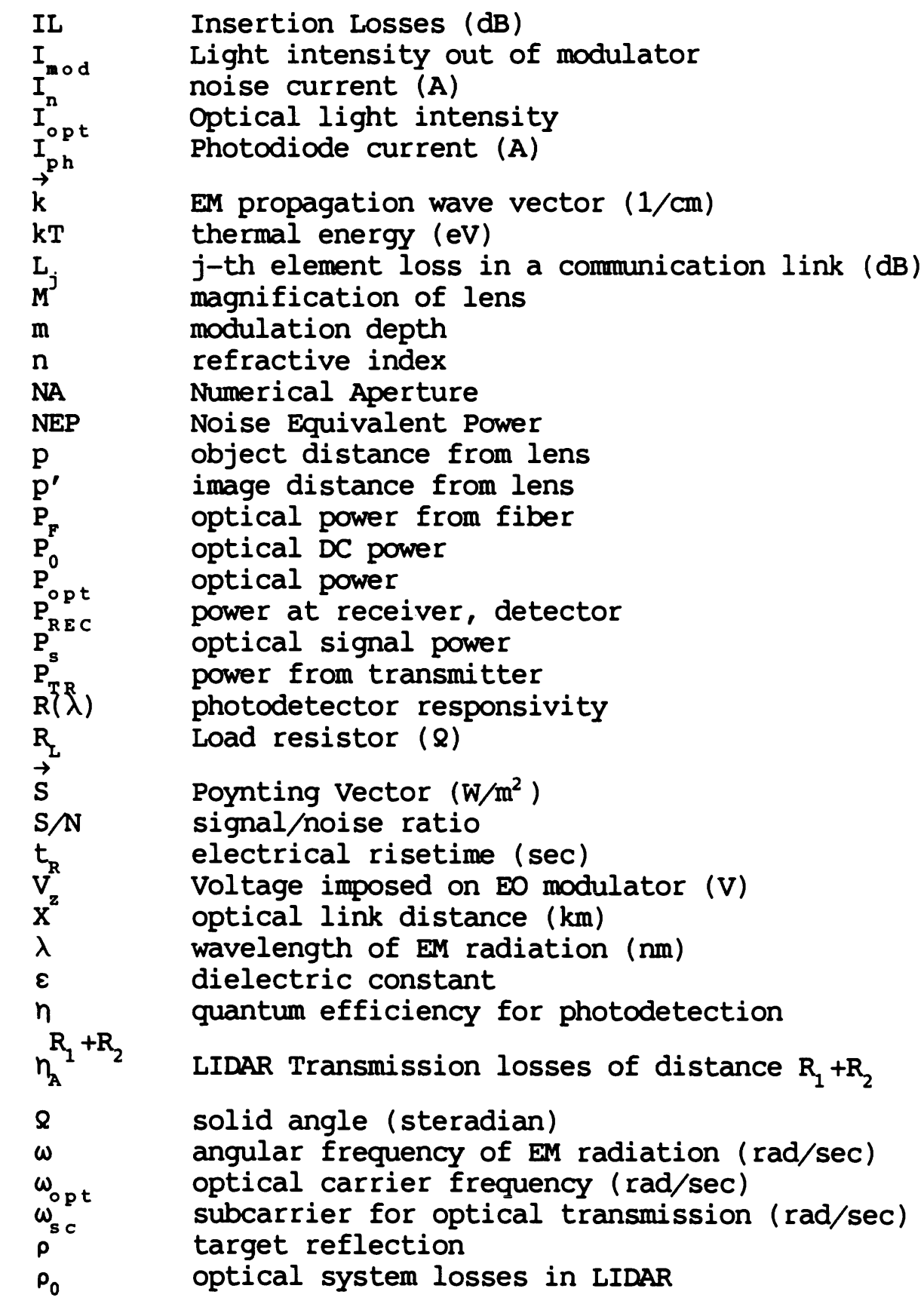

